\section{A Maximum of Temperature in the Middle Ionosphere}

J. Fuchs ${ }^{1}$ has already pointed out the fact that from the change of electron-density $(N)$ with height (h) conclusions as to the temperature in the ionosphere can be drawn. It was, however, not possible then to carry out exact calculations for two reasons : first, the electron-density was only known to be dependent on the apparent height $\left(h^{\prime}\right)$, and secondly, the process of olectron loss was not known in more detail, so that Fuchs had to calculate with a recombination. coefficient constant as to space and time.

To-day these two difficulties have been overcome; some institutions have for some time been publishing detailed $N(h)$ profiles, for example, the Central Radio Propagation Laboratory (Boulder) hourly values for the station Puerto Rico. For the process of electron loss, $I^{2}$ have introduced the following equation :

$$
L=\alpha_{9} p k \cdot T^{n} \cdot \mathrm{Nm}_{+}{ }^{2}
$$

It has been possible recently to define the constants $k$ and $n$ and this has ensbled me to determine from the $N(h)$ profiles the height dependency of scale height $(H)$ and density. The results with regard to the observations of Puerto Rico can be summarized as follows :

(1) The scale height has during the day a maximum at a height of about $200 \mathrm{~km}$. (2) The value of this maximum shows a considerably daily and yearly variation. In the early morning and late afternoon the maximum is much less noticeable than at noon. (3) The height in which this maximum occurs shows a corresponding daily and annual variation : at noon during the summer the maximum of $H$ rests at a height of about $220 \mathrm{~km}$.; it descends to about 180 $\mathrm{km}$. in the morning in winter. (4) Above this maximum the scale height decreases rap:dly, and $I$ presume that the gradient $\mathrm{d} H / \mathrm{d} h$ is getting small only a little below the F2 peak. (5) The decrease in density with height corresponds roughly to the result which Priester ${ }^{3}$ was able to publish recently.

This research is sponsored by the European Research Office (Contract No. 3983-S).

Institut für Meteorologie und Geophysik,

O. Burkard

(1936).

' Burkard, O., Geofis. pura e app., 87, 145 (1957).

"Priester, W., Mitteil. Univ. Sternuarte Bonn, No. $34 b$ (1960).

\section{GEOLOGY}

\section{Flow Folding in Rocks}

Prof. L. U. DE STTTER ${ }^{x}$ considers that in flow folding "internal movement itself is no longer orientated but can take any direction", and that the position of shear planes is random. 'He believes it developed most typically in incompetent rocks like salt, or at very high confining pressures or high temperatures. It is further maintained that this is the only true plastic deformation of rocks. These beliefs have recently been reiterated and enlarged on ${ }^{2}$.

Such views appear to be becoming general, and Ball $^{3}$ further characterizes monogenetic folds formed by flow by stating that "the quartz fabric will tend to be markedly heterogeneous, and the symmetry of the fabric will not correspond to the symmetry of the fold".
A brief account of the concepts on plastic flow of rocks will indicate that the current use of the term 'flow' has probably to do with only one type, which is difficult to accept as occurring within rocks.

Flow in rocks is a permanent change of form with. out apparent rupture, and any fracturing that may occur is microscopic. This property of flow is referred to as plasticity 4 . Above microscopic level such plastic flow is a continuous deformation where neighbouring points in the material remain so during the deformation.

Leith $^{5}$ and others have stated that flow takes place by interior readjustments of rock substances by chemical, mineralogical, and mechanical changes. Commonly, the deformation results in a parallel arrangement of the constituents of the rock mass, producing a banded structure, schistosity or cleav. age $^{6}$. However, flow may leave no evidence in any parallel arrangement.

Little is known of the mechanics of plastic flow. Nevertheless, possible comparisons between plastic and viscous flow can be made $e^{\prime}$ and the mechanics and the successful application of steady, stable, laminar flow to a number of folds have been described in pipers by Cloos ${ }^{8}$ and Carey ${ }^{8,10}$.

Folds that appear to have resulted from a laminar type of flow more or less perpendicular to the rock layers are characterized by thickened and thinned beds, crenulation of bedding planes, and generally a persistence in depth. Where planar discontinuities have developed and folding has progressed by movement along these planes the resulting deformation has been termed a shear fold.

It appears that these laminar-flow folds are excluded from de Sitter's class of flow folds which, because of the postulated random movement, must refer to a mechanism comparable with turbulent viscous flow.

According to reasonable calculations magma is intruded in most dykes by means of laminar flow and turbulence is only achieved in those magmas which have low viscosity due to super-heating or to a high content of volatile material11. By analogy it appears most unlikely that a type of turbulent flow can ever be achieved within rocks, which have, in comparison, enormously high viscosities.

It is, therefore, suggested that the folds at present believed to belong to de Sitter's class of flow folds should be reconsidered, and attempts made to inter. pret them in terms of laminar flow, for not all folds that result from laminar flow may appear geometrically simple. It has been proposed that lateral laminar flow along involuted planes within liquefied layers of sand can account for convolute folding that may occur within them ${ }^{12}$. Is it perhaps possible that the complicated plastic folds with, at first sight, random orientation of the foliations, are in point of fact due to similar unusual patterns of laminar flow?

It appears that the class of flow folds, the present status of which is here questioned, should be redefined to include folds resulting from laminar plastic flow. In so far as all rocks flow during folding, limits must necessarily be rather arbitrary. Competent layers, which retain approximately their original thickness perpendicular to the bedding, show only sufficient flow for them to be flexed. Thus, flexural or concentric folds, and folds of patterns determined by the flexing of competent layers within an interbedded sequence of competent and incompetent horizons would be omitted. Only those folds determined by
flow rather than flexure should be included. Flow 